\title{
DIÁLOGOS E INTERFACES DA ABORDAGEM GEOGRÁFICA NA EDUCAÇÃO PROFISSIONAL DE NÍVEL TÉCNICO EM SAÚDE
}

\author{
Maurício Monken \\ Fundação Oswaldo Cruz, Escola Politécnica de Saúde Joaquim Venâncio \\ mauriciomonken@gmail.com
}

\section{INTRODUÇÃO}

Os séculos $X X$ e $X X I$ empreenderam profundas transformações nos modos de viver de sociedades, resultado do intenso processo de globalização da economia com expansão e incorporação massiva de processos técnicos em redes de cadeias produtivas e de finanças em todos os lugares do planeta, com apropriação de recursos locais e intensificação de fluxos de circulação e de troca de informações, materialidades e pessoas (GONDIM e MONKEN, 2016).

A velocidade desses acontecimentos no espaço-tempo continua efetuando alterações profundas em territórios com consequências no ambiente, na vida social, na cultura, na economia e na política, as quais exigem respostas imediatas e singulares de setores governamentais para enfrentar os riscos e as vulnerabilidades introduzidas por meio de processos produtivos, de consumo e de circulação de bens e mercadorias. No campo da saúde, por exemplo, demandam ações para vigiar e prevenir problemas de saúde (riscos causas e danos) e dar assistência às diferentes populações - da cidade, do campo e da floresta e das águas.

Quando se dirige o olhar sobre as repercussões da globalização na saúde das populações, notam-se diversos aspectos preocupantes como a poluição ambiental, a disseminação das doenças infecciosas, os novos padrões comportamentais em saúde e não poderíamos deixar de mencionar a desorganização sociocultural dos lugares provocada pela instalação de grandes corporações multinacionais que afetam a determinação social da saúde nos territórios. Como ressalta Milton Santos (2001), a "globalização perversa produz cada vez mais desemprego crônico, fome e pobreza que aumentam exponencialmente novas e velhas enfermidades, assim como crescem males espirituais, morais, egoísmo, cinismo, corrupção" entre outros, são alguns destes aspectos.

Diante deste quadro, temos como perspectiva outras possibilidades de trabalho e de intervenção na saúde das populações. Uma proposta de base territorial e popular, com potencial de orientar formas de educação profissional em saúde, que incorpore simultaneamente a determinação social do processo saúde-doença e a promoção da saúde como eixo norteador de processos e ações (FERNANDES, 2018).

Se o propósito for transformar as práticas de saúde mediante a redefinição de políticas e a reorganização dos processos de trabalho, não se pode subestimar a questão pedagógica (PAIM; ALMEIDA, 2000) (MONKEN, 2008). No entanto, devemos considerar que a formulação de propostas para a educação profissional em saúde, muito embora possa representar importante colaboração para as mudanças almejadas nos processos de trabalho, sempre encontrará limitações dadas pela própria cultura institucional estabelecida nos sistemas de saúde na organização das práticas de saúde.

Para Paim e Almeida Filho (2000: 81), "a revisão e o desenvolvimento curricular podem ser medidas necessárias para a reatualização das instituições de ensino face à reorganização das práticas de saúde, porém insuficientes para alterar o modo de produção dos agentes".

A educação profissional é expressão recente, criada para designar processos históricos referentes à capacitação para (e no) trabalho. Aqui entendida como uma relação permanente entre o trabalhador e o processo de trabalho, assumindo caráter associado às ideias de autonomia e de autovalorização. Uma educação transformadora no sentido de superar a divisão social do trabalho, o que coloca a

Recebido em: 13/10/2019

Aceito para publicação em: 11/11/2019

Palestra proferida no IX Simpósio Nacional de Geografia da Saúde, Blumenau, SC, 2019.

página 83 
educação profissional e, em especial neste ensaio, de nível técnico, num contexto de lutas políticas e técnicas (PEREIRA, 2006).

Tem como propósito estabelecer diálogo entre categorias e métodos da geografia na educação profissional em saúde, no sentido de contribuir para um agir em saúde com base na determinação social da saúde e na promoção de territórios saudáveis e sustentáveis.

Para atingir os objetivos, apresentamos inicialmente as possibilidades da abordagem geográfica na educação profissional em saúde de nível técnico, tendo como centralidade o processo de globalização que vem alterando profundamente os modos de vida e a saúde das populações nos territórios. Destacamos a seguir a categoria território e o processo de territorialização como estratégia pedagógica para contextualizar a situação de saúde nos e como eixo central para a formulação dos currículos nos diversos processos formativos de nível técnico. Ao final faz uma discussão sobre a aplicação desta abordagem nos processos formativos na área de atuação da Vigilância em Saúde da Escola Politécnica de Saúde Joaquim Venâncio da Fundação Oswaldo Cruz (EPSJV/Fiocruz).

\section{EDUCAÇÃO PROFISSIONAL EM SAÚDE E GEOGRAFIA: IDENTIFICANDO RELAÇÕES}

A educação dos profissionais de saúde no Brasil e no mundo tem como base uma concepção em que os aspectos biológicos e o desenvolvimento técnico-científico são privilegiados nos currículos em detrimento das questões de ordem social, política, cultural e ética. O principal enfoque na formação está nos componentes biológicos, na doença e no trabalho clínico e hospitalar, produzindo uma excessiva especialização e o distanciamento dos conteúdos curriculares em relação ao perfil do profissional da saúde e às necessidades sociais da população. (ALBUQUERQUE; GIFFIN, 2008).

As contribuições da abordagem geográfica para este debate são significativas, principalmente no que se referem as suas possibilidades de contextualizar pedagogicamente a realidade social no processo formativo dos trabalhadores. Possibilita desvendar as relações de produção e sociais concretas, de superar o existente, contidos nas contradições geradas pelo embate capital $x$ trabalho, promovendo condições para a emancipação do trabalhador, seja em sua relação com o pensamento, seja em relação a ordem social e econômica imposta. (GONDIM; MONKEN, 2016)

Este movimento faz com que outros campos de conhecimento, como da geografia, por meio de algumas de suas categorias centrais, expressem a "totalidade dos estados das coisas e das situações existentes" (SANTOS, 1999), considerando a realidade em sua integridade, como totalidade social, com potencial para produzir uma formação contextualizada e pedagogicamente possuidora de sentido para o trabalhador.

O objetivo é educar para outro mundo possível, para o fortalecimento do pensamento crítico na formação dos profissionais de saúde fornecendo ferramentas epistêmicas para que consigam refletir sobre sua própria prática, disputar um projeto de sociedade e lutar conscientemente pela consolidação do Sistema Único de Saúde no Brasil, comprometido com a universalidade, a equidade e a integralidade do cuidado. (ALBUQUERQUE; GIFFIN, 2008)

São variados os aspectos em que a análise geográfica pode contribuir na formação de profissionais de saúde. $\mathrm{Na}$ atualidade, categorias e metodologias advindas da geografia são recursos indispensáveis para conhecer as condições e vida e a situação de saúde de territórios, os determinantes e necessidades sociais de saúde, riscos e danos. Estas categorias ressurgem nas últimas décadas, como resposta ao forte processo de globalização e seus efeitos socioambientais, principalmente a partir da questão local como contraponto a mundialização dos vetores sociais, econômicos, culturais e políticos inerentes a esse processo.

A dimensão local e seus modos de vida necessitam ser compreendidos e observados não só como lócus de moradia e depositário final de eventos, mas sobretudo como lugar de produção e reprodução social, de trocas materiais e simbólicas e de convivência entre pessoas. (GONDIM; MONKEN, 2018) 
O uso do conhecimento geográfico, no campo da saúde, com a finalidade de identificar e localizar elementos do lugar e da população para compreender a influência e contribuição na ocorrência de eventos de saúde é recente, embora seja antiga a sua incorporação em vários momentos da história da saúde pública. (GONDIM; MONKEN, 2018)

A partir da questão local de reprodução social, faz com que a escala dos acontecimentos e de produção da vida, se destaque como categoria e como uma das formas iniciais de compreensão dos fenômenos sociais. A partir da definição da escala de análise de observação, as outras categorias geográficas se associam em uma rede de conceitos e de metodologias, para serem utilizadas não só no estudo como na formação de profissionais de saúde sobre a realidade social e seus contextos.

O geógrafo Antonio Carlos Robert de Moraes (2011) ressalta que cada escala e cada conceito geográfico se adéquam ao trato de determinados fenômenos e problemas. A visualização das situações e o equacionamento dos problemas derivam de opções escalares e conceituais. O que é evidente para um olhar local pode se perder numa leitura regional e vice-versa. A escolha das escalas a serem utilizadas tanto nos diagnósticos territoriais quanto no desenho dos objetivos $e$ procedimentos de políticas é fundamental na estruturação de uma abordagem geográfica, se constituindo em elemento essencial para sua perspectiva de êxito, que diz respeito às divisões do espaço assumidas e as respectivas unidades espaciais a serem trabalhadas.

Os efeitos dos vetores da globalização, principalmente por meio das novas tecnologias de comunicação e informação tem tido papel crucial não somente na produção e seus efeitos locais nos contextos de vida, como propiciando a articulação entre os diversos atores sociais na resolução dos problemas de saúde. Os atores sociais do Estado (em seus diversos setores de atuação - saúde, educação outros) como das empresas locais e das corporações multinacionais, dos movimentos sociais e de organizações não governamentais tem hoje papel decisivo na produção dos contextos de vida, seus problemas e potencialidades, sejam estas influências de origem regional, nacional ou mundial que, cada vez mais, estão presentes no dia a dia dos lugares.

Após definir a escala de observação, o primeiro e talvez o mais evidente papel das categorias geográficas, seja fornecer, como mencionamos acima, um quadro contextualizado das condições de vida nos espaços, territórios e lugares onde será aplicada qualquer forma de observação ou política de atuação. Ẻ da tradição do campo da geografia a elaboração de diagnósticos delimitados geograficamente, abordando tanto suas características naturais quanto as alterações derivadas de seu processo de apropriação, produção e uso. (MORAES, 2011)

Espaço, categoria central na Geografia, foi a primeira a ser instrumentalizada pelo setor saúde. Inicialmente na Epidemiologia, para evidenciar o local onde a doença e a morte de indivíduos e populações ocorriam, observando causas e riscos relacionados. Em seguida, foi incorporado à organização e gestão dos serviços para identificar necessidades de saúde e compreender processos (acesso, deslocamentos, alocação de recursos), fluxos (de pessoas, de serviços, de insumos, profissionais, agravos) e mobilidade populacional.

Conceituado como pedaço de terra modificado ao longo da história pela organização social, pelas técnicas e pela economia, o espaço geográfico é socialmente produzido e palco de realizações humanas com as características de ser produzido pelos grupos sociais; como possuidor de dinamicidade própria - as ações humanas mudam constantemente; ter uma forma fragmentada e organizada em recortes espaciais; apresentar singularidade - é apropriado de diferentes formas; e ser multiescalar - abrangência local, regional, nacional, global. (GONDIM; MONKEN, 2018)

Outro conceito fundamental na formação de trabalhadores da saúde, em especial nos processos de trabalho para atuar na contextualização de diagnósticos territoriais, é a noção de 'lugar'. Conceito definido como espaço percebido relacionado a códigos e valores e à dimensão afetiva ou de identidade ou a local onde se tem familiaridade ou intimidade (uma rua, uma praça, uma área de atuação ou a própria casa) é utilizado para designar proximidade e pertencimento territorial e para identificar os processos de representação dos territórios pelos atores locais. (GONDIM; MONKEN, 2018). 
A região é outra categoria de uso em diversas áreas de atuação da saúde, como na Gestão de Sistemas de Saúde, na Vigilância e na Atenção Primária em Saúde. Conceito que expressa maior ou menor proximidade e maior ou menor amplitude territorial significando formas de percepção do território, um espaço dividido que obedece a critérios específicos e elaborado para especificar determinada área ou aspecto dela.

As regiões podem ser criadas para fins de estudo sobre características gerais de um território (regiões brasileiras) ou para destacar determinado aspecto (regiões geoeconômicas, agrupadas segundo perfil econômico). São limites territoriais estabelecidos para fins de conhecer e atuar sobre as condições de vida e a situação de saúde de populações, que não devem interferir na territorialização existente, definindo critérios para a governança das regiões de saúde, de ordem organizacional e operacional, adequados às características socioculturais e sanitárias, dos equipamentos públicos e dos serviços de saúde existentes no território, no sentido de inclusão e de acesso a segmentos sociais que tenham identidade em relação aos limites regionais. (GONDIM; MONKEN, 2018)

Associado ao conceito de espaço, o 'território' vem sendo usado nos últimos 20 anos de múltiplas formas no campo da saúde. No Brasil foram fortes as influências das propostas de Atenção Primária em Saúde, que resultou em processo de inserção territorial de unidades de saúde na organização de serviços de saúde que por sua vez propiciou a redefinição do próprio conceito de território nas políticas públicas de saúde, dando lugar a propostas mais amplas do que a de território político administrativo, conferindo-Ihe maior densidade teórico-metodológica.

Muito além de ser meramente um recorte espacial, em se tratando de pesquisa social e humana, refere-se ao processo de territorialização na apropriação e ocupação pelas pessoas e grupos dos mais diversos, numa dinâmica de interação social que apresenta diferentes contextos de poder de uso político dos recursos pelos atores no território e consequentemente de condições de vida e situação de saúde.

Nos processos de territorialização, relações de poder são estabelecidas por meio de trocas, de diálogos, de negociações, de pactuações ou de conflitos entre diferentes pessoas e grupos que, em dado momento, propõem implementar localmente seus projetos ou intervenções. Portanto, poder como essência das relações sociais de apropriação sobre o espaço geográfico, é conceito central para o entendimento de território. (GONDIM; MONKEN, 2018; MONKEN; GONDIM, 2016)

As organizações sociais, culturais, religiosas, lazer, o Estado, a população, as empresas e outros atores possuem poder e o exercem de acordo com seus interesses e projetos e com suas capacidades de fazer com que ele aconteça e se materialize nos territórios. (MONKEN; GONDIM, 2016). São diversos atores que se apropriam e fazem uso político do território impondo suas regras. Já outros, atuam a distância de acordo com as novas possibilidades tecnológicas, mas que cada vez mais, interferem nos modos de vidas da população.

Todos esses movimentos de territorialização em conjunto geram conflitos de interesses que surgem das relações, ou seja, materializam-se em disputas entre os diversos grupos para organizar por meio de pactuações, o território, da maneira mais adequada aos objetivos de cada um. (GONDIM; MONKEN, 2018).

Portanto, território não somente é um dos princípios organizativo-assistenciais mais importantes do sistema de saúde, como é base conceitual de uma proposta metodológica aplicada às práticas de saúde denominada de "processo de territorialização de informações em saúde".

\section{O PROCESSO DE TERRITORIALIZAÇÃO COMO ESTRATÉGIA PEDAGÓGICA NA EDUCAÇÃO PROFISSIONAL DE NÍVEL TÉCNICO EMM SAÚDE}

Conceito recentemente desenvolvido, territorialização expressa um processo definido que traduz um fenômeno social. Um ato de estar, agir, fazer ou fixar no espaço geográfico delimitando um território. Um processo de apropriação que tem como base a capacidade dos atores sociais de exercer algum DOI:http://dx.doi.org/10.14393/Hygeia153351679 Hygeia 15 (33): 83 - 90, Set/2019 página 86 
tipo de poder de ação que propicie construir identidade, regras, vínculos, normas e ordenamento territorial (GONDIM; MONKEN, 2017).

Atores sociais (pessoas, empresas e instituições) ao se estabelecerem em lugares, territorializam suas histórias, seus hábitos, suas normas, seus costumes, seus recursos individuais, seus projetos, seus desejos, definindo contextos específicos e as formas de apropriação e uso dos espaços. É um movimento de encontro no território, de um conjunto de elementos semelhantes e diversos, que em interação vão contribuir (positiva ou negativamente) para a produção social da saúde ou da doença. Esse processo possibilita a criação de identidade e de pertencimento da população com os espaços do cotidiano (GONDIM; MONKEN, 2017; 2018).

O processo de territorialização pode ter três dimensões que se apresentam de forma simultânea. A partir do momento que é utilizado para organizar as práticas de trabalho de forma territorializada, se configura como um método de pesquisa-ação e como uma estratégia pedagógica em processos formativos.

Originalmente no setor saúde, compõe uma das ferramentas básicas de atuação da Vigilância em Saúde e dá suporte ao planejamento estratégico situacional e a programação de ações que deve ser desenvolvida pela equipe de saúde local e pela comunidade como pilares de sustentação das ações nos territórios para o reconhecimento das condições de vida e da situação de saúde.

A territorialização enquanto método de pesquisa se constrói pela busca sistemática de informações sobre um território por meio de metodologias quantitativas, qualitativas e técnicas de investigação de campo, produzindo e revelando conhecimento sobre realidades sócio-sanitárias-espaciais (GONDIM; MONKEN, 2003), estabelecendo um rico diálogo com a realidade social, seus sujeitos, cenários e situações.

Enquanto estratégia pedagógica articula-se com a pesquisa sobre as condições de vida e a situação de saúde, onde a produção da informação deve ser entendida como um insumo para que se dê o desenvolvimento da aprendizagem. No interior desse processo, em função das mediações que se estabelece entre os saberes e as práticas cotidianas ressignifica a aprendizagem.

É uma técnica essencialmente pedagógica, pois ensina a melhor forma de estabelecer políticas conforme especificidades identificadas. Um processo de manifestações e interações com a realidade social, que compartilha a criação de conhecimento, pois para além dos dados acumulados, pode levar à reformulação das observações preconcebidas por meio da aprendizagem e descoberta de outros caminhos.

Determinadas técnicas de pesquisa da Geografia se destacam nos processos de territorialização a serem incorporados ao trabalho territorializado em saúde e em processos de educação profissional para a saúde pública.

O mapeamento seja na forma manual ou digital, é um instrumental historicamente estabelecido na Geografia que dá representação a materialidade embutida nos processos de territorialização como fenômeno social. Informações territorializadas em mapeamento podem desvelar relações associativas e evidenciar interações entre elementos do território imbricados nas condições de vida de populações. Como representação da realidade percebida contribui para elucidação da determinação social de eventos de saúde. Não podemos encarar o método cartográfico como meramente descritivo, como pode parecer, mas sim como instrumento capaz de possibilitar interpretações, relacionamentos e montar cenários. (GONDIM; MONKEN, 2018)

A técnica de entrevista de campo com atores sociais locais, tradicional forma de pesquisa das ciências humanas, quando associada às categorias geográficas que expressem a apropriação, representação e uso político dos espaços pelos atores locais, possibilita conhecer a história de ocupação e uso do território, as regras e normas e o pacto social envolvido na convivência espacial cotidiana, os problemas e necessidades percebidos e as potencialidades que podem ser acionadas para resolução de problemas, identifica organizações sociais e sua capacidade de ação local - do 
poder público e em especial do setor saúde, de entidades civis, de movimentos sociais e de lideranças comunitárias.

Técnica muito aplicada pela Geografia e com potencial de utilização na educação profissional é a produção de imagem associada ao método observacional. Utilizados em conjunto, ampliam oportunidades de aprendizagem sobre a apropriação, representação e uso político pelos atores sociais dos recursos do território, potencializando pedagogicamente o reconhecimento territorial e as melhores escolhas na tomada de decisão para ação. (MONKEN, 2008)

\section{CATEGORIAS GEOGRÁFICAS COMO DISPOSITIVOS PARA APRENDIZAGEM NA EDUCAÇÃO PROFISSIONAL DE NÍVEL TÉCNICO EM VIGILÂNCIA EM SAÚDE}

Nos últimos 20 anos as experiências de formação realizadas na EPSJV/Fiocruz possibilitaram introduzir categorias e métodos geográficos em currículos de cursos com foco na interface existente entre as condições de vida e a situação de saúde.

A abordagem do território e da territorialização no Curso Técnico de Vigilância em Saúde da EPSJV para trabalhadores que desenvolvem ações de campo, se concretizou de forma transversal aos currículos de modo a relacionar conteúdos, temas e eixos, por entender que é no território onde se materializa a vida cotidiana e suas articulações com a totalidade social e assim desenvolver as práticas de saúde.

Conteúdos temáticos e estratégias pedagógicas que introduzem a categoria território e métodos da geografia na formação do trabalhador da vigilância em saúde expressam as formas que os atores sociais, individual ou coletivo, público ou privado, utilizam para influenciar ou controlar pessoas, recursos, fenômenos e relações, delimitando e efetivando a apropriação, representação, uso e o poder sobre uma área, comunidades, bairros, municípios, estados e até mesmo países (GONDIM; MONKEN, 2016).

Os conceitos e as categorias geográficas, utilizadas nos processos formativos da EPSJV/Fiocruz, contribuíram para descrever e entender as formas de viver e se relacionar de pessoas e grupos, conhecer seus "modos de levar a vida" e as relações que estabelecem entre si e com o espaço produzido, ou seja, como produzem, se apropriam e representam os espaços e ambientes para poder viver, se reproduzir, realizar relações e trocas materiais e simbólicas (GONDIM; MONKEN, 2016).

Trabalhadas nos processos formativos em trabalho de campo e em sala de aula, têm possibilitado a aprendizagem sobre o reconhecimento de subjetividades, emoções e memórias locais e identificado problemas, necessidades e positividades dos lugares, contribuído para tomada de decisão e definição de estratégias de ação nas múltiplas dimensões do processo de saúde-doença-cuidado.

Por meio da estratégia pedagógica do trabalho de campo, efetuado por meio do processo de territorialização das condições de vida e situação de saúde, propõe-se articulação sistemática entre teoria e prática, ensino-serviço-comunidade. O trabalho pedagógico desenvolvido nos cursos em diversos territórios do Sistema Único de Saúde (SUS) com organizações sociais e instituições atuantes nesses espaços, favorece a aprendizagem, o diálogo e a parceria para efetivação dos princípios e diretrizes do SUS, destacando a relevância e o papel da população na produção social de saúde.

A aplicação desta técnica desenvolve a aprendizagem possibilitando desde o conhecimento do lugar da produção social da saúde, como espaço de identidade do trabalhador, seguindo pela investigação de situações no nível local, como espaço de articulação institucional deste trabalhador, permitindo a análise da situação de saúde e das condições de vida; como espaço de decisão do trabalhador, de poder de ação e, neste circuito educativo; como espaço de ação do trabalhador, no entendimento da natureza mais adequada para a intervenção sobre problemas de saúde e necessidades sociais. (GONDIM; MONKEN, 2016) 
Associado à territorialização, o trabalho de campo é um procedimento didático e estratégia pedagógica que em movimento e de forma simultânea constrói conhecimento e educa. Reconstrói saberes teóricos propostos no processo formativo, no cotidiano de seu trabalho e de sua vida. Uma maneira de aprender e de ensinar, por articular teoria e prática e ensino, serviços e comunidade, que amplia a autonomia do estudante e do professor como um exercício de cidadania.

\section{CONSIDERAÇÕES FINAIS}

No contexto brasileiro, o setor saúde tem sido marcado, em sua história recente, pela organização de um modelo centrado no aspecto clínico e assistencial, resultando na ampliação de um complexo industrial da saúde globalizado dominado por grandes corporações internacionais. Este modelo não tem respondido de forma efetiva aos problemas de saúde de populações em países com características demográficas, epidemiológicas e sanitárias como o Brasil, marcados por desigualdades sociais e de dificuldade de acesso aos cuidados de saúde (GONDIM, 2011).

Outras formas atuação podem contribuir para reverter este quadro. As categorias geográficas discutidas neste ensaio - escala, espaço geográfico, lugar e região quando associadas conceitualmente dão suporte teórico à técnicas de pesquisa tradicionais desta ciência que possibilitam o olhar sobre o processo de adoecimento de forma contextualizada e a partir das especificidades dos modos de vida dos lugares.

São categorias e métodos que tornam cada vez mais evidentes maneiras de organizar os processos de trabalho e de formação, conduzindo não somente as ações do Estado e da prática cotidiana dos movimentos sociais, como também da ciência como uma possibilidade teórica e metodológica para estudos e pesquisas.

"Fazer falar o território" (SANTOS, 2001) é premissa para pensarmos em formas de trabalho em saúde que, por meio de um processo ou método organiza as práticas que situa o território de atuação para cumprir com a integralidade do cuidado e a participação da comunidade nos processos decisórios, como técnica de reconhecimento de realidades sociais, de escuta e diálogo que envolve decisões de parte a parte.

Em sua dimensão pedagógica faz com que na educação profissional a territorialização seja o dispositivo educacional que dá 'voz ao lugar' e escuta o território como caminho simultâneo para apreensão e aprendizagem dos contextos de vida e saúde observados. Uma tecnologia estruturante que de forma simultânea, torna-se meio de ensino e trabalho em suas diferentes formas de apropriação.

\section{REFERÊNCIAS BIBLIOGRÁFICAS}

ALBUQUERQUE, V S; GIFFIN, K F. Globalização capitalista e formação profissional em saúde: uma agenda necessária ao ensino superior. Trabalho, Educação e Saúde. Rio de Janeiro: 2008, vol.6, n.3, pp.519-538. https://doi.org/10.1590/S1981-77462008000300007

FERNANDES, VR; MONKEN, M; GONDIM, GMM et al. Denaturalizing "Long-Lasting Endemic Diseases": social mobilization in the context of arboviral diseases in Brazil. In SALAZAR, LM; VILLAR, RCL (org.) Globalization and Health Inequities in Latin America. Editora Springer, 2018. https://doi.org/10.1007/978-3-319-67292-2 5

GONDIM, G; MONKEN, M. Saúde, Educação, Cidadania e Participação: a experiência do Proformar. Trabalho, Educação e Saúde. Rio de Janeiro: Editora da EPSJV/FIOCRUZ, v. 01(02), p. 35-39, 2003.

GONDIM, GMM; MONKEN, M. Geografia e Saúde no âmbito da Educação Profissional Técnica de Nível Médio: 20 anos formando trabalhadores para o SUS. In MAGALHÃES, SC; PEREIRA, MPB 
(org). Pesquisa e extensão em Geografia da Saúde: entre a teoria e a prática. Montes Claros: Unimontes, 2017. https://doi.org/10.7476/9788575416297.0008

GONDIM, G; MONKEN, M. O Uso do Território na Atenção Primária à Saúde. In Atenção Primária à Saúde no Brasil: conceitos, práticas e pesquisa. MENDONÇA, MHM et al (org.). Rio de Janeiro: Editora Fiocruz, 2018.

MONKEN, M; GONDIM, G.M.M. Território: Lugar onde a vida acontece. In: BORSTEIN, VJ. (Org). Curso de Aperfeiçoamento em Educação Popular em Saúde: Textos de Apoio. Rio de Janeiro: EPSJV, 2016.

MONKEN, M. Contexto, Território e Processo de Territorialização de Informações: desenvolvendo estratégias pedagógicas para a educação profissional em saúde. In BARCELLOS, C (org.). A Geografia e o Contexto dos Problemas de Saúde. Rio de Janeiro: ABRASCO; ICICT; EPSJV, (Saúde e Movimento; n. 6), 2008.

MORAES, ACR. Territorialização. Cadernos temáticos para o panorama do saneamento básico no Brasil. In REZENDE, SC. (org.). Brasília: Ministério das Cidades/Secretaria Nacional de Saneamento Ambiental, 2011.

PAIM JS; ALMEIDA, NAF. A crise da saúde pública e a utopia da saúde coletiva, Vol. 1. Coleção de Saúde Coletiva. Salvador: Casa da Qualidade Editora, 2000.

SANTOS, M. A natureza do espaço: técnica e tempo, razão e emoção. São Paulo: Editora Hucitec; 2001. 